\title{
Pressure-sensitive adhesive combined with paper spray mass spectrometry for trace surface detection of illicit drugs and explosives (Withdrawal Notice)
}

Daniel Carmany, Chao Bao Nguyen, Trevor Glaros, Nicholas Manicke, Elizabeth Dhummakupt

Daniel Carmany, Chao Bao Nguyen, Trevor Glaros, Nicholas Manicke, Elizabeth Dhummakupt, "Pressure-sensitive adhesive combined with paper spray mass spectrometry for trace surface detection of illicit drugs and explosives (Withdrawal Notice)," Proc. SPIE 11749, Chemical, Biological, Radiological, Nuclear, and Explosives (CBRNE) Sensing XXII, 1174909 (12 April 2021); doi: 10.1117/12.2585936 


\title{
Pressure-sensitive adhesive combined with paper spray mass spectrometry for trace surface detection of illicit drugs and explosives (Withdrawal Notice)
}

\author{
Daniel Camany \\ Exc et, Inc. (United States) \\ Chao Bao Nguyen and Nicholas Manicke \\ Zhejiang Univ. (China) \\ Trevor Glaros \\ India na Univ.-Purdue Univ. Indianapolis (United States) \\ Elizabeth Dhummakupt \\ CCDC Chemical Biologic al Ctr. (United States)
}

Proceedings Volume 11749, Chemic al, Biologic al, Radiological, Nuc lear, and Explosives (C BRNE) Sensing XXIl; 1174906 (2021) https:// doi.org/10.1117/12.2585936

Event SPIE Defense + Commercial Sensing Digital Forum, 2021, O nline Only, United States

Online Publication Date: 12 April 2021

Withdrawn from Publication: 5 May 2021

Publisher's Note: This paper, orig ina lly published on 12 April 2021, was withdrawn per author request. 